Proceedings

\title{
Enhancing the Performance of Commercial Infrared Detectors by Surface Plasmons ${ }^{\dagger}$
}

\author{
Kirsi Tappura \\ VTT Technical Research Centre of Finland Ltd., FI-33101 Tampere, Finland; kirsi.tappura@vtt.fi; \\ Tel.: +358-40-704-1773 \\ + Presented at the Eurosensors 2018 Conference, Graz, Austria, 9-12 September 2018. \\ Published: 16 November 2018
}

\begin{abstract}
The feasibility to improve the response of quantum type (photonic) infrared (IR) detectors by applying surface plasmons is investigated. The $\mathrm{HgCdTe}$ material system is used as the detector platform of interest for which selected plasmonic structures and materials are applied and the influence studied by full-fielded electromagnetic simulations. It is shown that even for the severalmicrometers-thick detector structures, similar to the commercial ones, broadband absorption enhancements of $30-40 \%$ can be achieved. The results suggest that improved, or new, pixel-level functionalities can be created for commercial IR detectors by relatively simple means. Additionally, high potential for cost reduction in high-performance IR imaging systems with multicolour capabilities is foreseen.
\end{abstract}

Keywords: infrared detectors; plasmonics; computational simulations; finite element method

\section{Introduction}

Over the past several years, the research on exploiting surface plasmons for photon management has experienced a remarkable growth in various application fields, such as thin-film solar cells, nearfield optical microscopy, subwavelength wave-guiding, molecular sensing and photodetectors $[1,2]$. Conventionally the main efforts have been focused on the visible or near-infrared part of the spectrum. However, there is a growing interest towards plasmonics for longer wavelengths due its high potential for enhancing the performance of infrared detectors [3] e.g., in thermal imaging and chemical sensing. Especially, the safety and security applications impose many critical challenges on future high-end infrared detectors and imaging cameras that are not fully met by the available commercial IR systems. In addition to the constant demand for better performance (sensitivity, thermal resolution, pixel density etc.), improvements in the usability of the high-end sensors are desired (smaller size, less cooling).

\section{Materials and Methods}

Numerical simulations are performed to investigate the coupling of infrared radiation into $\mathrm{HgCdTe}$ based detectors. The device structure under study imitates a basic design of a longwavelength infrared detector that includes an $\mathrm{Hg}_{1-\mathrm{x}} \mathrm{Cd} \mathrm{C}_{\mathrm{x}} \mathrm{Te}(\mathrm{x} \approx 0.2)$ absorber of several micrometers think sandwiched between CdTe layers (Figure 1). Plasmonic structures prepared of gold, silver, and copper are assembled on the top CdTe layer of 30-50 nm in thickness.

The three-dimensional (3D) full-wave Maxwell equations are solved using the finite element method (FEM by Comsol® Multiphysics) with perfectly matched layer (PML) boundary conditions. Plane waves propagating perpendicular to the surface of the detector are used for excitation. A rectangular periodic lattice is formed by applying periodic boundary conditions (PBC, see Figure 1). 
The material parameters (i.e., dispersive permittivity) of $\mathrm{HgCdTe}, \mathrm{CdTe}$, and metals were obtained from refs. [4-6], respectively.

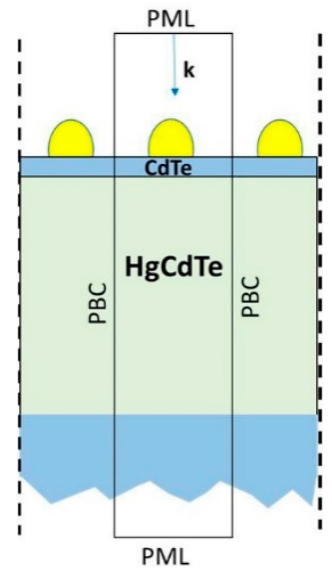

Figure 1. A cross sectional view of the simulated system with plasmonic structures (yellow) on the thin CdTe passivation layer on the top of the IR absorber. The wave vector, $\mathbf{k}$, indicates the direction of the incident plane wave and the sold rectangular box the simulation box, on the vertical sides of which the periodic boundary conditions (PBC) are applied.

The time-average spectral power density, $Q_{a b s,}$ absorbed in volume $V$ is used to calculate the power absorbed in the detector structure:

$$
Q_{a b s}(\omega)=\frac{\omega}{2} \int_{V} \operatorname{Im}[\varepsilon(\omega)]|\boldsymbol{E}|^{2} d V
$$

where $E$ is the electric field, $\omega$ the angular frequency of the electromagnetic radiation, and $\varepsilon(\omega)$ the permittivity of the material in volume $V$.

\section{Results}

In the present work, plasmonics structures are developed for standard $\mathrm{HgCdTe}$ infrared detectors for the spectral range of about $8-12 \mu \mathrm{m}$ by computational means. The influence of selected plasmonic geometries and materials on the device performance is evaluated. As demonstrated in Figure $2 \mathrm{a}$, broadband absorption enhancements of $30-40 \%$ are feasible with the proposed geometries even for the standard detector structures with several-micrometers-thick absorbers. The plasmonmodified interactions of the infrared radiation with the detector can be tuned as shown in Figures 2 and 3 , as examples.

The demonstrated beneficial effects are created by engineering the scattering properties of the plasmonic structures so that most of the incident radiation is scattered into the absorber in favorable angles to enhance the optical path of photons in the active layer and to reduce the reflection losses similar to an efficient broad-band antireflection layer. In addition, the electromagnetic near-field is enhanced near the plasmonic structures resulting to confined and increased absorption near the structures in the absorber. As expected, for thinner absorbers the effects are more pronounced. Figure $2 \mathrm{~b}$ shows the relative scattering cross section of a single gold particle similar to the ones used in the array of the upper curve of Figure 2a indicating that the absorption in the plasmonic particles can be minimized and the scattering into the detector maximized. 


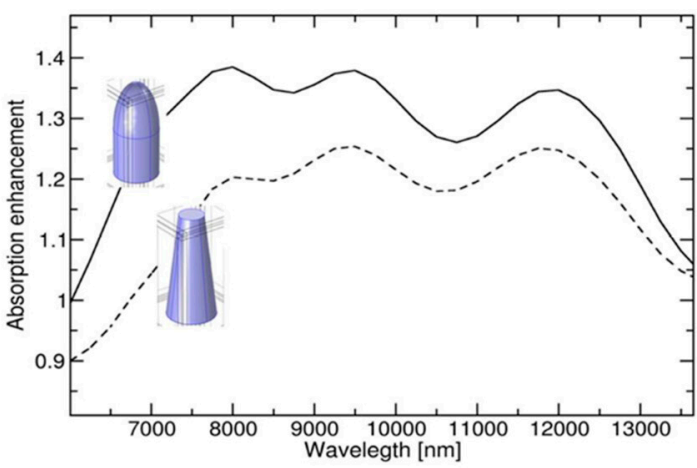

(a)

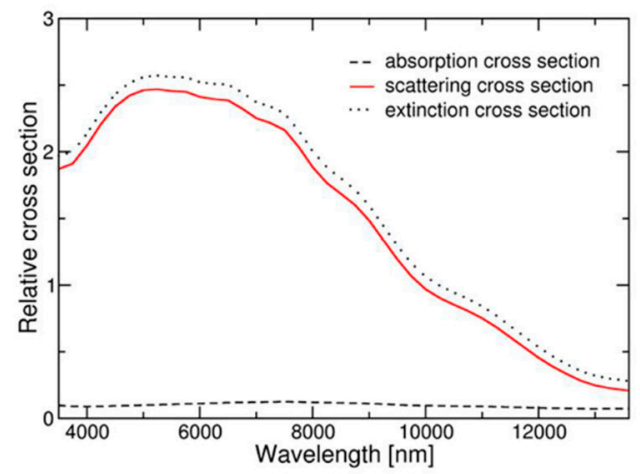

(b)

Figure 2. (a) Influence of the shape of the plasmonic structures on the absorption enhancements in an $\mathrm{HgCdTe}$ infrared detector with a 6-um-thick absorber. The absorption enhancement is defined as the ratio of the power absorbed in $\mathrm{HgCdTe}$ for the device with plasmonic structures to that without the plasmonic structures; (b) Absorption, scattering and extinction cross sections relative to the geometrical cross section for a single plasmonic particle similar to the ones used in the array of the upper curve of Figure 2a.

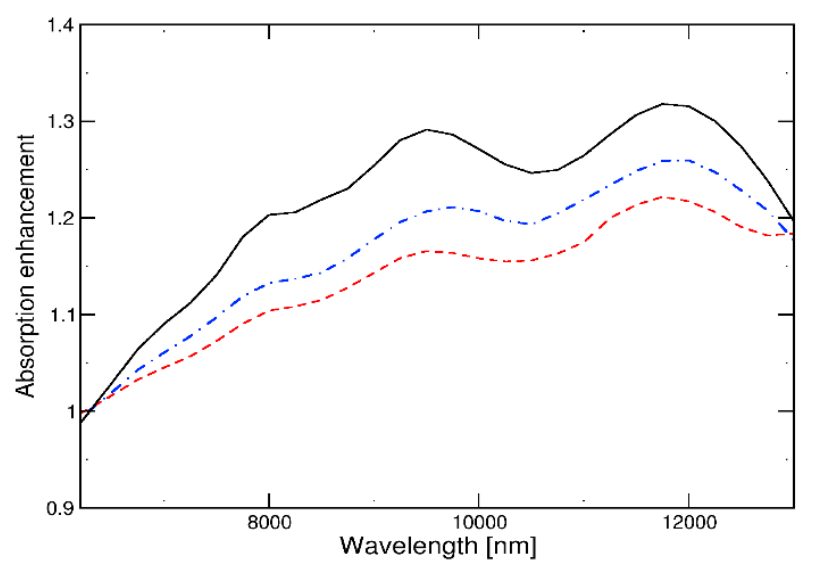

Figure 3. Absorption enhancement of a thick $\mathrm{HgCdTe}$ detector tuned by varying the surface density of the metallic plasmonic structures. The absorption in $\mathrm{HgCdTe}$ increases as the coverage increases from $9 \%$ (red dashed line) to $18 \%$ (black solid line).

In addition to designing detectors with enhanced absorption and photoresponsivity, the spectral response ("color") can also be engineered with accordingly designed plasmonic structures by enhancing selected wavelength regions and suppressing the others. Further, the results suggests that properly designed plasmonic structures provide the means to decrease the volume of a detector/pixel and as such to decrease the noise levels, as well.

\section{Discussion}

Regardless of the increasing number of reported results and predictions, the true potential of plasmonic structures for enhancing the performance of different IR detectors is still largely undefined and even more, not exploited in products. Nevertheless, the reported results suggest that plasmonics can provide a promising practical tool for the designers of future IR detectors with enhanced pixel level functionalities.

Considering the fast development of high-throughput and high-resolution fabrication techniques the advancement of plasmonic structures even into relatively low-cost devices seems feasible. A significant advantage of the plasmonics approach, as demonstrated here, is that the 
plasmonic structures - even the ones based on metallic patterns - can be introduced into a standard IR detector or imaging camera fabrication process without major additional efforts.

\section{Conclusions}

In spite of the widely available commercial photonic and thermal detectors, there is still a need especially for high-end IR imaging systems that combine an excellent performance, reasonable price and convenient operation (i.e., less cooling), while the lower-end detectors lack a convenient, costeffective method to improve the thermal resolution. Inclusion of application-specific plasmonic designs into the fabrication processes of infrared detectors is foreseen as a promising path towards both the targets.

Author Contributions: K.T. conceived, designed and performed the simulations; K.T. analyzed the data; K.T. contributed analysis tools; K.T. wrote the paper.

Acknowledgments: This project has received funding from the Finnish Scientific Advisory Board for Defence (MATINE) and partially from VTT Technical Research Centre of Finland Ltd.

Conflicts of Interest: The author declares no conflict of interest. The founding sponsors had no role in the design of the study; in the collection, analyses, or interpretation of data; in the writing of the manuscript, and in the decision to publish the results.

\section{References}

1. Schuller, J.A.; Barnard, E.S.; Cai, W.; Jun, Y.C.; White, J.S.; Brongersma, M.L. Plasmonics for extreme light concentration and manipulation. Nat. Mater. 2010, 9, 193-204, doi:10.1038/nmat2630.

2. Tappura, K.; Luomahaara, J.; Haatainen, T.; Hassel, J.; Vehmas, T. Influence of substrate on plasmoninduced absorption enhancements. Plasmonics 2016, 11, 627-635, doi:10.1007/s11468-015-0090-4.

3. Jakšić, Z;; Milinović, M.; Randjelović, D. Nanotechnological enhancement of infrared detectors by plasmon resonance in transparent conductive oxide nanoparticles. J. Mech. Eng. 2012, 58,367-375.

4. Pautrat, J.L.; Magnea, N. Optical Absorption in HgCdTe and related heterostructures. In Properties of Narrow Gap Cadmium-Based Compounds; EMIS Data Reviews Series No. 10, ed. P. Capper; IEE: London, UK, 1994; pp. 75-79.

5. DeBell, A.G.; Dereniak, E.L.; Harvey, J.; Nissley, J.; Palmer, J.; Selvarajan, A.; Wolfe, W.L. Cryogenic refractive indices and temperature coefficients of cadmium telluride from $6 \mu \mathrm{m}$ to $22 \mu \mathrm{m}$. Appl. Opt. 1979, 18, 3114-3115.

6. Rakić, A.D.; Djurišic, A.B.; Elazar, J.M.; Majewski, M.L. Optical properties of metallic films for verticalcavity optoelectronic devices. Appl. Opt. 1998, 37, 5271-5283.

(c) 2018 by the authors. Licensee MDPI, Basel, Switzerland. This article is an open access article distributed under the terms and conditions of the Creative Commons Attribution (CC BY) license (http://creativecommons.org/licenses/by/4.0/). 